\title{
Efficacy of Nd:YAG Laser for the Treatment of Hidradenitis Suppurativa
}

\author{
Neda Nosrati ${ }^{*}$, Lindsey Hunter, Erica Kelly \\ Department of Dermatology, University of Texas Medical Branch, Galveston, USA. \\ Email: *nenosrat@utmb.edu
}

Received March 21 ${ }^{\text {st }}, 2012$; revised April 25 ${ }^{\text {th }}, 2012$; accepted May $6^{\text {th }}, 2012$

\begin{abstract}
Hidradenitis suppurativa (HS) is an inflammatory skin disease that involves sebaceous glands and hair follicles of intertriginous areas, including axillary and inguinal regions. It is often chronic, painful, disabling, and can worsen over time. Besides surgically excising affected areas, effective treatments are currently lacking. The long-pulsed neodymium:yttrium-aluminium-garnet (Nd:YAG) laser is a device that has traditionally been used for hair removal, but has recently shown promise in clearing old lesions of HS and preventing new eruptions. In accordance with studies in the current literature, we hypothesize that the Nd:YAG laser will significantly improve subjects with hidradenitis suppurativa compared to standard, uninvasive treatment with topical antibiotics [1,2]. Study aims include: to treat subjects with hidradenitis suppurativa with Nd:YAG laser therapy once monthly for 3 consecutive months, with a one month followup; to compare the effectiveness of Nd:YAG treatment on one side of the subject (e.g., right) to treatment with topical antibiotics on the subject's other side (e.g., left); to assess treatment response using a visual analogue scale; to assess subject satisfaction with treatment using a questionnaire; and to photodocument treatment response at each visit.
\end{abstract}

Keywords: Hidradenitis Suppurativa; Nd:YAG Laser; Acne Inverse; Follicular Disease

\section{Introduction}

Hidradenitis Suppurativa (HS) is a recurrent and chronic inflammatory disease which can be disfiguring to patients. Usually patients present with tender inflammatory abscesses of the flexures, including the axillary, inframammary, and inguinal areas. Scarring and tract formation are prominent features. Females seem to be more often affected than males. Patients are usually post pubertal. The chronic and scarring nature of the disease often leads to significant impacts on the quality of life in these patients [1,2].

HS was first described by Velpeau, a French physician, in 1839 . He was also the first to hypothesize that the disease was related to purulent inflammation of the sweat glands, later thought to be mainly inflammation of apocrine [2]. More recent data however, supports the theory of follicular occlusion as the predominant feature with apocrine involvement a secondary feature. Bacterial colonization is also likely a secondary event which may exacerbate disease. Other factors which may be associated, but not etiologic, include smoking and obesity [2].

The prevalence of HS is estimated at about $1 \%$ of the population, but unfortunately there are limited effective treatment options [3]. Furthermore, large-scale rando-

"Corresponding author. mized controlled trials have not been extensively performed. Current treatment options include topical or systemic antibiotics, isotretinoin, dapsone, biological agents, and surgical interventions. Increasing interest in the use of biologics has led to several recent studies, albeit with only limited numbers of patients. Infliximab was shown to be effective in small studies; however, its use is limited by both adverse effects and cost. Adalimumab, according to a recent review, has not shown consistent results supporting its use despite its growing popularity although it has been well tolerated with no serious side effects reported in any of the studies. Traditional surgical intervention has been excision of the affected areas with variable recurrence rates and high associated morbidity. Newer approaches include the $\mathrm{CO}_{2}$ and Nd:YAG lasers both with promising results [4].

A study performed by Tierney et al in HS patients with moderate disease showed a $65.3 \%$ improvement in severity of disease after 4 monthly treatments with Nd:YAG laser. They theorized that the hair follicle was the nidus for inflammation and that destruction of the follicle would lead to improvement. They were able to support this theory with histopathologic evidence that the laser worked through selective photothermolysis of the follicular unit with a reduction in inflammatory lesions [1]. 
Based on the success of this study and others like it recently in the literature, we decided to further explore the efficacy of the Nd:YAG laser in treatment of patients with HS. Our study was unique in that we recruited patients even with minimal disease as the Nd:YAG laser has a very high safety profile with essentially no risk to the patients; therefore, justifying its use in even minimal disease to prevent scarring and tract formation. Our goals were to treat patients with HS with Nd:YAG laser therapy once monthly for 3 consecutive months and reevaluate after one month, to compare the effectiveness on one side (treatment side) of the patient to the other side (control side), to assess treatment response using a visual analogue scale, to assess subject satisfaction with a subject questionnaire, and to photodocument treatment response at each visit.

\section{Methods}

Six patients ( 5 women and 1 man) ages 19 to 54 carrying a clinical diagnosis of hidradenitis suppurativa were enrolled initially in this study, performed at the University of Texas Medical Branch in Galveston, Texas from February 2011 through February 2012. The study was approved by the UTMB institutional review board and was in accordance with the Declaration of Helsinki. A sample size of 5 was calculated to have $80 \%$ power to detect a difference in means of 23.400 (e.g. a First condition mean, $\mu_{1}$, of 31.000 and a Second condition mean, $\mu_{2}$, of 7.600), assuming a standard deviation of differences of 12.000 , using a paired $t$-test with a 0.050 two-sided significance level.

Patients were included if they had at least Hurley stage I disease of their axilla (abscess formation without sinus tracts and cicatrization) [5]. Patients were excluded for the following reasons: pregnancy, previous surgical treatment to affected areas, or any concomitant systemic therapy. Patients on systemic therapy were allowed to enter the study as long as they discontinued the therapy at least 2 weeks prior to the initiation of the study laser protocol.

First the treatment side (right vs. left axilla) was chosen using a computerized randomization tool. Three monthly laser treatments were then performed using a single pass of the long pulsed 1064-nm Nd:YAG laser (Cutera Inc., Brisbane, California) with a one month follow up after completion of the three treatments, for a total study period of four months. A topical cooling gel was employed using the standard laser treatment protocol, but no anesthetics were used. A contact metal-tipped cooling device was used on the treatment areas immediately before and after irradiation. The laser settings were chosen based on the patient's Ftizpatrick skin type per Cutera's recommendations. Patients were given an aftercare instructions handout. Topical 1\% Clindamycin gel was used on both the control and treatment sides. After the study period of 4 months, patients were allowed to continue receiving treatment to the treatment side and initiate treatment to the control side at no cost if they so desired.

Patients were contacted within 48 hours of treatment to assess for adverse events. Patients were also evaluated at the follow up visits by the treating physician for any development of adverse events, including pigmentary alterations.

Photos were taken of treated and untreated sides with and without flash prior to each session and at the one month follow up. Photographs were then evaluated by both a blinded physician and resident. Initially the modified HS LASI score designed by Sartorius et al. [6] was to be the evaluation tool; however, it was difficult to use this scoring system with the photographs, and a new modified score was created by our center based on the Sartorius score (Table 1).

In addition, patients were asked to fill out a subject assessment questionnaire after each treatment visit. The questionnaires assessed the patients' overall satisfaction with the treatment and their subjective improvement as well as assessing for any side effects of therapy.

A paired $t$-test was done to compare the mean percentage of change for both treated and control sites for each patient from baseline to the fourth month. Of note the scores between the physician scorer and resident scorer were not different based on t tests; therefore, we averaged across scorers. The $p$ value was calculated for the mean percentage of change and a value of less than 0.05 indicates statistical significance for the study.

\section{Results}

A total of 5 patients completed this 4 month study. One patient (19 year old female) withdrew prior to beginning the study secondary to scheduling conflicts. The mean age was 37.6 years, with $80 \%$ females $(n=4)$ and $20 \%$ males $(n=1)$. Fitzpatrick skin types ranged from II to V, with 3 patients having type II-III, and one patient each having type IV and type V. Only axillary disease was treated in this study.

Table 1. Modified scoring system based on Sartorius et al.

\begin{tabular}{lll}
\hline $\begin{array}{c}\text { Abscess/Nodule } \\
\text { Formation }\end{array}$ & \multicolumn{1}{c}{$\begin{array}{c}\text { Tract } \\
\text { Formation }\end{array}$} & $\begin{array}{c}\text { Scar } \\
\text { Formation }\end{array}$ \\
\hline 0-not present & 0-not present & 0-not present \\
1-mild activity & 1-mild activity & 1-mild activity \\
2-moderate activity & 2-moderate activity & 2-moderate activity \\
3-severe activity & 3-severe activity & 3-severe activity \\
\hline
\end{tabular}

Based on 3 factors, including abscess/nodule formation, tract formation, and presence of scarring. Highest possible score is 9 indicating more severe disease, while a score of 0 indicates no active disease. 
The mean score at baseline for the treatment sites was 2.4 (SD: 0.33) which increased to 2.75 (SD: 2.56) at the end of the 4 months compared to the mean score at baseline for the control sites which was 3.05 (SD: 1.89) and increased to 3.15 (SD: 2.77) at the end of the 4 months (Figure 1). There was no statistical significance in the percentage of change in the scores after 4 months of treatment $(\mathrm{P}=0.41)$. This is likely related to the fact that the power to detect statistical significance was very limited due to the small sample size of 5 patients.

Before and after photographs are demonstrated in Figure 2. None of the patients reported experiencing any side effects when they were contacted shortly after each treatment. All patients opted to continue laser treatments after the 4 month study period was completed.

The subject assessment questionnaire was distributed to the patients at the end of each treatment session and at the end of the study. Overall, $80 \%$ of patients $(n=4)$ rated their overall level satisfaction with the laser treatment as very satisfied. Only one patient $(20 \%)$ rated their level of satisfaction as unsatisfied. None of the patients reported having any pain associated with treatment or any other side effects including redness, swelling, crusting or changes in skin pigmentation. Patients were also encouraged to leave comments which included the following: "less outbreaks and leaking from boils" and "no new breakouts," "the [treatment] side had no problems at the beginning of the study and remains so. The [control] side has developed a new nodule."

\section{Discussion}

Although it appears logical to assume that inflammation of apocrine glands is the primary event in HS as the lesions are distributed mainly in apocrine rich locations, recent evidence actually suggests that involvement of the follicle and thus the pilosebaceous unit is the primary event and apocrine gland involvement is a secondary phenomenon. Studies based on histopathology describe a series of events similar to the pathogenesis of acne with hyperkeratinization of the follicle leading to dilation and rupture causing dermal inflammation and bacterial colonization, sinus tract formation, and eventually scarring [7]. These similarities between the two disorders have led to the suggestion of acne inversa as a more appropriate description than HS [8]. Furthermore, studies have shown that similar to acne toll-like receptor 2 expression is increased in macrophages and dendritic cells of patients with HS [9]. However, there are several differences which exist between acne and HS including microbiology, clinical presentation, and response to treatment [7]. Overall, there is still no absolute consensus as to the etiology of HS, but the hair follicle likely plays an integral role. Localization to intertriginous areas may be explained by shear forces in these areas leading to micro tears in the hair follicle, which is exacerbated by larger skin folds in the obese [10].

The long pulsed Nd:YAG laser is a device that has been traditionally used for hair removal. This treatment has the advantage of being strictly local and devoid of systemic side effects such as those encountered with administration of systemic steroids, antibiotics, retinoids, and biologics. Furthermore, the long pulsed Nd:YAG is safe even in darker skin types due to its longer wavelength and greater depth of penetration with less effects on the epidermis leading to pigmentary alterations [11].

As discussed previously, the treatment options for HS are limited and none have proven to be dramatically effective [4]. Few of the biologics and surgery have shown

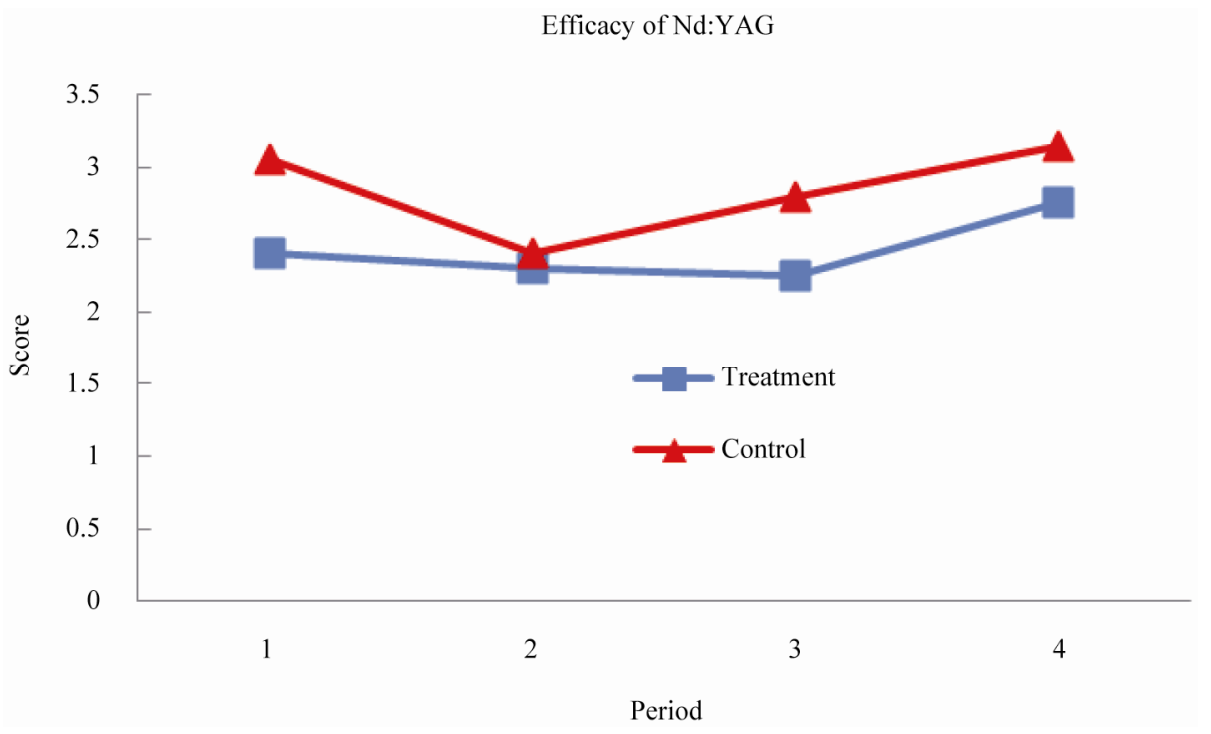

Figure 1. Mean scores across treatment periods from baseline to final visit at 4 months. 


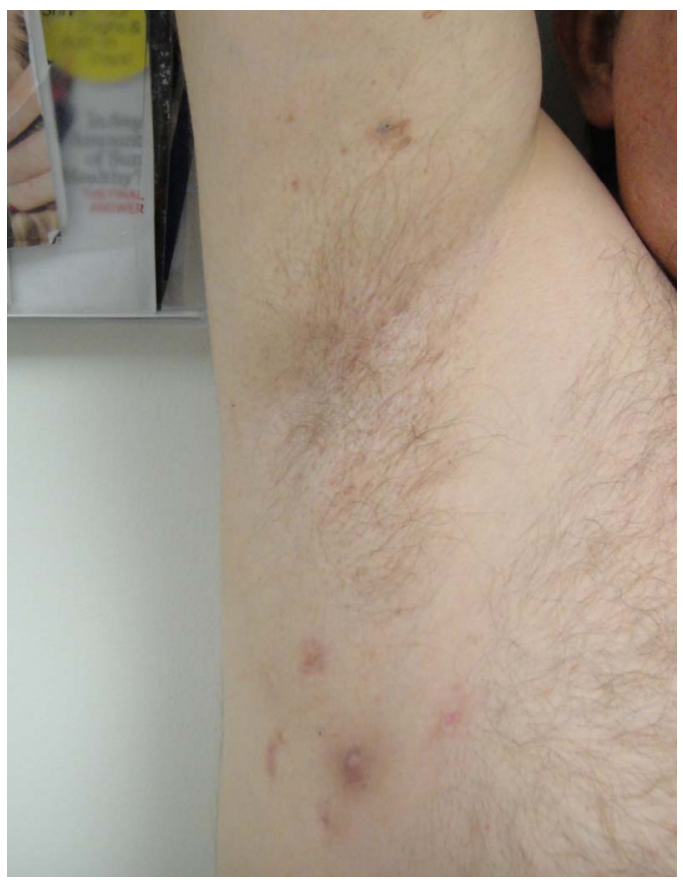

(a)

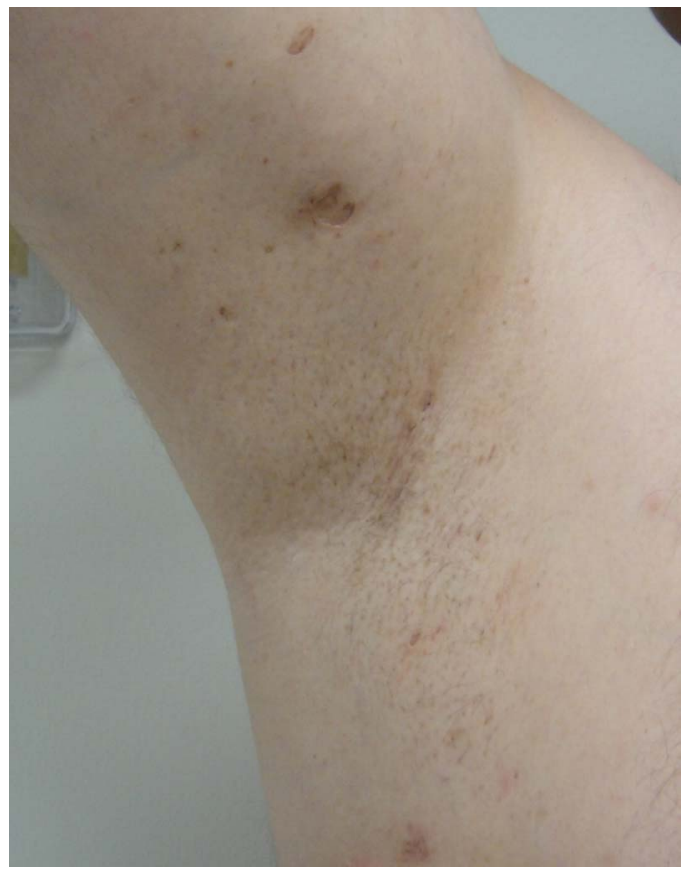

(b)

Figure 2. (a) Immediately before initial treatment showing small abscesses and mild scarring; (b) One month follow up after 3 consecutive monthly treatments shows decreased inflammatory lesions.

some improvement, but are associated with a high rate of adverse events. Nd:YAG laser is safe and effective for hair epilation and as recent scientific evidence points to the hair follicle as the primary abnormality in HS; it seems logical to assume that Nd:YAG should be effec- tive as a treatment of HS.

Unfortunately, our results did not show a significant difference in the treatment side versus the patients' untreated side (control) after 3 consecutive monthly treatments with the Nd:YAG laser. This is likely due to the very small sample size of only 5 patients. There was a limited time period allowed for the completion of this study after IRB approval was finally obtained, which did not allow for ample recruitment time. Had the sample size been larger, there may have been a demonstrable response to the laser as has been seen in several other similar studies evaluating the Nd:YAG in HS $[1,3]$.

Another factor which likely influenced the non significant results was the scoring system used. Initially the Sartorius Score was to be the visual analog scale used to score the photographs. It was decided to use photographs rather than actually evaluating the patient in person to allow for blinding of the evaluator. However, the physician scorers found it very difficult to adequately judge distances between two relevant lesions which was required by the Sartorius score; therefore, the scoring system had to be adjusted. This new scoring system may have led to poor scoring data and thus nonsignificant results.

Despite the fact that the results were not significant, the patients seemed overall pleased with the results with $80 \%$ rating their satisfaction level as very satisfied. Furthermore, the one patient who rated their experience as unsatisfied, chose to drive over 4 hours round trip to our clinic once a month for continued treatment with the laser. Actually, all the patients chose to continue laser treatment after the study period for several months with many of the patients driving very long distances. No side effects were noted by any of the patients during the entire study period with most of the patients tolerating very high fluences up to $70 \mathrm{~J} / \mathrm{cm}^{2}$.

In conclusion, patients were overall satisfied with $\mathrm{Nd}: Y A G$ laser treatment for HS and continued to request therapy even though no statistical difference between treatment and control sites was objectively observed. Furthermore, Nd:YAG laser was found to be completely safe with no side effects encountered throughout the study even when using high fluences. Therefore, laser is a safe alternative in patients with even mild disease in HS, although we cannot prove that it is more effective than topical antibiotics alone.

\section{REFERENCES}

[1] L. Y. Xu, D. R. Wright, B. H. Mahmoud, D. M. Ozog, D. A. Mehregan and I. H. Hamzavi, "Histopathologic Study of Hidradenitis Suppurativa Following Long-Pulsed 1064 nm Nd:YAG Laser Treatment," Archives of Dermatology, Vol. 147, No. 1, 2011, pp. 21-28. doi:10.1001/archdermatol.2010.245 
[2] A. Alikhan, P. J. Lynch and D. B. Eisen, "Hidradenitis Suppurativa: A Comprehensive Review," Journal of the American Academy of Dermatology, Vol. 62, No. 4, 2009, pp. 706-708.

[3] B. H. Mahmoud, E. Tierney, C. L. Hexsel, J. Pui, D. M. Ozog and I. H. Hamzavi, "Prospective Controlled Clinical and Histopathologic Study of Hidradenitis Suppurativa Treated with the Long Pulsed Neodymium: YttriumAluminium-Garnet Laser," Journal of the American Academy of Dermatology, Vol. 62, No. 4, 2010, pp. 637-645. doi:10.1016/j.jaad.2009.07.048

[4] P. V. Rambhatla, H. W. Lim and I. Hamzavi, "A Systematic Review of Treatments for Hidradenitis Suppurativa," Archives of Dermatology, Vol. 148, No. 4, 2011, pp. 439446. doi:10.1001/archdermatol.2011.1950

[5] H. Hurley, "Dermatologic Surgery, Principles and Practice," Marcel Dekker, New York, 1989.

[6] K. Sartorius, J. Lapins, L. Emtestam and G. B. Jemec, "Suggestions for Uniform Outcome Variables When Reporting Treatment Effects in Hidradenitis Suppurativa," British Journal of Dermatology, Vol. 149, No. 1, 2003, pp. 211-213. doi:10.1046/j.1365-2133.2003.05390.x

[7] S. Kamp, A. M. Fiehn, K. Stenderup, C. Rosada, B. Parkkenberg, K. Kemp, T. N. Dam and G. B. Jemec, "Hidradenitis Suppurativa: A Disease of the Absent Sebaceous Gland? Sebaceous Gland Number and Volume
Are Significantly Reduced in Uninvolved Hair Follicles from Patients with Hidradenitis Suppurativa," British Journal of Dermatology, Vol. 164, No. 5, 2011, pp. 1017 1022. doi:10.1111/j.1365-2133.2011.10224.x

[8] J. Boer and E. F. Weltevreden, "Hidradenitis Suppurativa or Acne Inversa: A Clinicopathologic Study of Early Lesions," British Journal of Dermatology, Vol. 135, No. 5, 1996, pp. 721-725. doi:10.1111/j.1365-2133.1996.tb03880.x

[9] R. E. Hunger, A. M. Surovy, A. S. Hassan, L. R. Braathen and N. Yawalkar, "Toll-Like Receptor 2 Is Highly Expressed in Lesions of Acne Inversa and Colocalizes with C-Type Lectin Receptor," British Journal of Dermatology, Vol. 158, No. 4, 2008, pp. 691-697. doi:10.1111/j.1365-2133.2007.08425.x

[10] H. Kurzen, I. Kurokawa, G. B. Jemec, L. Emtestam, K. Sellhever, E. J. Giamarellos-Bourboulis, I. Nagy, F. G. Bechara, K. Sartorius, J. Lapins, D. Krahl, P. Altmeyer, J. Revuz and C. C. Zouboulis, "What Causes Hidradenitis Suppurativa?" Experimental Dermatology, Vol. 17, No. 5, 2008, pp. 455-456. doi:10.1111/j.1600-0625.2008.00712.x

[11] T. S. Alster, H. Bryan and C. M. William, "Long Pulsed Nd:Yag Laser-Assisted Hair Removal in Pigmented Skin: Clinical and Histological Evaluation," Archives of Dermatology, Vol. 13, No. 7, 2001, pp. 885-889. 